\title{
Protocol
}

\section{Synthesizing evidence on nutrition challenges among people living with HIV/AIDS in sub- Saharan Africa: a protocol for a scoping review}

\author{
Tafadzwa Dzinamarira ${ }^{1,2} *$, Moreblessing Chipo Mashora ${ }^{1}$
}

\author{
${ }^{1}$ Department of Public Health, Mount Kenya University, Kigali, Rwanda \\ ${ }^{2}$ ICAP, Mailman School of Public Health, Kigali, Rwanda
}

Received: 18 April 2019

Accepted: 08 June 2019

\section{*Correspondence:}

Mr. Tafadzwa Dzinamarira,

E-mail: anthonydzina@gmail.com

Copyright: (C) the author(s), publisher and licensee Medip Academy. This is an open-access article distributed under the terms of the Creative Commons Attribution Non-Commercial License, which permits unrestricted non-commercial use, distribution, and reproduction in any medium, provided the original work is properly cited.

\begin{abstract}
Background: Good nutritional status is highly significant for individuals who are infected with HIV. However, they still face a number of nutritional challenges. The proposed scoping review will map literature on the nutritional challenges facing people living with HIV/AIDS (PLWH) and guide future research in nutritional management to improve health outcomes for PLWH. Here we outline a scoping review protocol designed according to the Preferred Reporting Items for Systematic Reviews and Meta-Analysis Protocols (PRISMA-P 2015 Guidelines).

Methods: The Arksey and O'Malley's 2005 scoping methodological framework further improved by Levac et al. 2010 will guide the search and reporting. Searches will be conducted for eligible articles from MEDLINE (PubMed), MEDLINE, CINAHL, Academic Search Complete and ISI Web of Science (Science Citation Index) electronic databases. Two independent reviewers will conduct the search guided by an inclusion and exclusion criteria. Quality appraisal of the included articles will be conducted guided by the mixed methods appraisal tool 2018 version. We will employ NVivo version 12 for thematic content analysis.

Conclusions: The findings of this review will guide future research in nutritional management to improve health outcomes for PLWH in sub-Saharan African. This review will be disseminated electronically in a published peer reviewed article and in print.
\end{abstract}

Keywords: Nutrition challenges, People living with HIV/AIDS, Sub-Saharan Africa

\section{INTRODUCTION}

Over 37 million individuals are living with HIV across the globe as of 2017. Good nutritional status is highly beneficial for people living with HIV (PLWH). In most cases, HIV attacks the immune system of the people. ${ }^{1,2}$ Whereas good nutrition is one of the critical factors for PLWH, there are different kinds of nutrition challenges that are experienced by the PLWH. The scoping review will therefore map evidence on the main nutritional challenges faced.
Improper nutrition makes PLWH not to be in a good chance to maintain life, which is very healthy and of high quality. HIV infection largely damages the immune system of individuals, and this often results in other kinds of infections, such as fever and diarrhea. ${ }^{3,4}$ These also have a ripple impact of lowering intake of food as well as interfering with the body's capacity to absorb food. Hence, the individual becomes weakened, malnourished, and as a result loses a lot of weight. ${ }^{5,6}$ It can therefore be pointed out that nutritional care and support are the integral elements of any action taken. Despite the fact that there are various benefits that are associated with proper nutrition for the PLWH, within the sub-Saharan 
Africa, various challenges are always faced that result in malnutrition among PLWH.,

\section{METHODS}

\section{Identifying relevant studies}

Eligibility of the research question for a scoping review

Our research question is: What is the evidence on nutritional challenges among PLWH in sub-Saharan Africa? We have used the population, concept and context (PCC) framework to determine the eligibility of our research question for our scoping review study (Table $1)$.

Table 1: Framework for determining eligibility for research question.

\begin{tabular}{|ll|}
\hline Population & $\begin{array}{l}\text { Human participants; living with } \\
\text { HIV/AIDS }\end{array}$ \\
\hline Concept & $\begin{array}{l}\text { Any nutrition challenges reported among } \\
\text { people living with HIV/AIDS between } \\
\text { January 2000 to March 2019 }\end{array}$ \\
\hline Context & Sub-Saharan Africa \\
\hline
\end{tabular}

\section{Search strategy}

A comprehensive search of electronic databases will be conducted for peer reviewed journal articles. A threestage screening process, which consists of a title, abstract screen as well as a full-text review, will be used for determination of eligibility of the articles. For an article to be included, it must report evidence on nutrition challenges among PLWH in sub-Saharan Africa. Two reviewers will screen articles for eligibility. Discrepancies at abstract screen will be settled by discussion. A third reviewer will settle any discrepancies at full-text review.

\section{Information sources}

The Arksey and O'Malley's scoping methodological framework, 2005 with improvement by Levac et al will guide this review. ${ }^{7,9} \mathrm{We}$ will search electronic databases MEDLINE (PubMed), EBScoHost (MEDLINE, CINAHL and Academic Search Complete) and ISI Web of Science (Science Citation Index).

We have conducted a pilot search in PubMed to demonstrate feasibility of answering our research question using a scoping review method. The results of our pilot search are presented in Table 2.

Table 2: Results of pilot search in PubMed.

\begin{tabular}{|c|c|c|c|}
\hline Keywords Search & Date of search & $\begin{array}{l}\text { Search engine } \\
\text { used }\end{array}$ & $\begin{array}{l}\text { Number of publications } \\
\text { retrieved }\end{array}$ \\
\hline $\begin{array}{l}\text { ((sub[All Fields] AND Saharan[All Fields] AND } \\
\text { ("Africa"[MeSH Terms] OR "nutrition"[All } \\
\text { Fields]) AND challenge[All Fields]) AND } \\
\text { ("people"[MeSH Terms] OR "living"[All Fields])) } \\
\text { AND (("with"[MeSH Terms] OR ("HIV"'[All } \\
\text { Fields] AND "AIDS"[All Fields]) }\end{array}$ & $1 / 03 / 19$ & PubMed & 148,922 \\
\hline
\end{tabular}

\section{Study selection}

A selection criteria was developed in order to ensure that relevant studies on nutrition challenges among PLWH will be included.

\section{Inclusion criteria}

To be included articles must meet the following criteria:

- Reporting evidence on nutrition challenges among PLWH in sub-Saharan Africa.

- $\quad$ Published between January 2009 to February 2019.

\section{Exclusion criteria}

We will exclude studies guided by the following exclusion criteria:
- Studies reporting evidence of nutrition challenges among PLWH in middle-upper and high income countries.

- Studies published before January 2009 and after February 2019.

Table 3: Data charting form.

Author and publication year

\begin{tabular}{|l|}
\hline Study title \\
\hline Aim of study \\
\hline Total participants \\
\hline Age of participants \\
\hline Geography (where was the study area) \\
\hline Study design \\
\hline Most relevant findings \\
\hline Other findings \\
\hline
\end{tabular}




\section{Data extraction}

Two reviewers will screen the publications, discuss the results and amend the data charting form table (Table 3) before beginning screening for this review. Discrepancies between reviewer's data extraction results will be resolved by a third reviewer. Information will be extracted from the included articles using the data charting form (Table 3). The form will be constantly updated in an iterative process according to the data extracted and to fully answer the research question

\section{Collating, summarising and reporting results}

Consistent with Arksey and O'Malley's framework, we will extract data related to our research question using content thematic analysis approach. ${ }^{7,8}$ All data regarding nutrition challenges for PLWH in sub-Saharan Africa will be extracted from the included articles. This will be done by transforming those sentences into more precise data for classification, by tracking the main concepts and the papers that provided the evidence of such challenges. Any differences in interpreting the content of papers or their underlying themes will be resolved through a third reviewer. This is in line with the iterative nature of all the stages in scoping reviews. ${ }^{9}$

Steps to be taken are summarized in Figure 1.

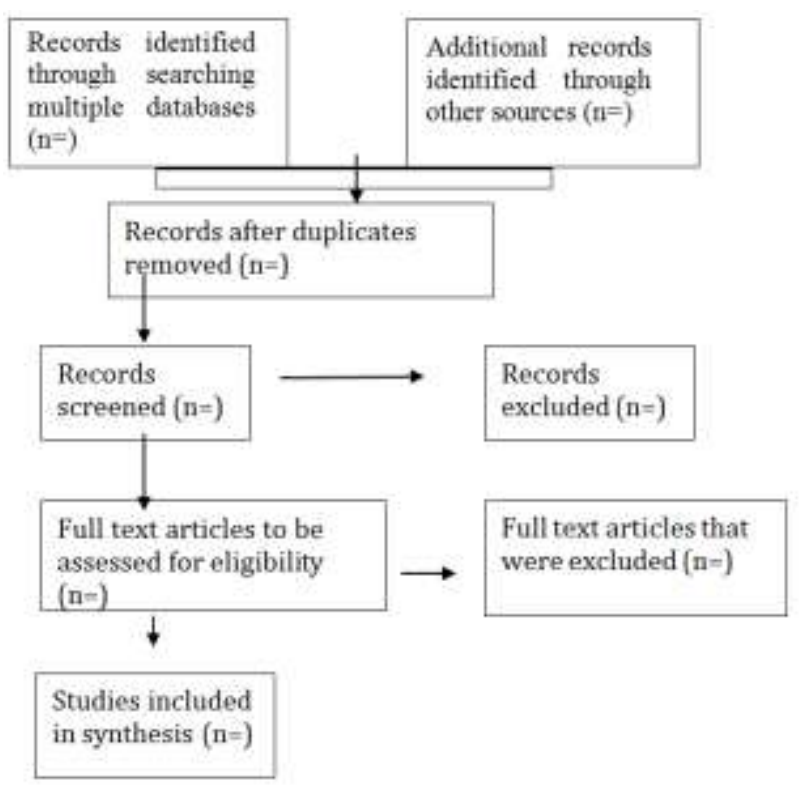

Figure 1: PRISMA extension for scoping reviews, 2018 flow diagram. ${ }^{12}$

\section{Quality appraisal}

The mixed method appraisal tool (MMAT) Version 2018 will be used to assess the quality of the included articles. ${ }^{10}$ The tool will be used to examine the aim of the study, adequacy and methodology, study design, participant recruitment, data collection, data analysis, presentation of findings, authors' discussions and conclusions. The MMAT was first developed in 2007 by Pluye et al, and was revised in more recently by Hong et al. ${ }^{10,11}$ The MMAT has been useful in appraising the quality of primary research. ${ }^{10,11}$ Two reviewers will independently carry out the critical appraisal process

\section{CONCLUSION}

The scoping review will offer a broad overview of the nutrition challenges among PLWH in sub-Saharan Africa. The results will be highly beneficial in pointing out some of the main nutritional challenges among PLWH in sub-Saharan Africa. In addition, the results will help identify gaps in the approaches, which are used to address the challenges being faced, offer better comprehension of the progress made and provide required support for implementation. At the same time, it will guide future research in nutritional management to improve health outcomes for PLWH.

\section{ACKNOWLEDGEMENTS}

The authors would like to extend their appreciation to Dr. Tivani Phosa Mashamba- Thompson from College of Health Sciences, University of KwaZulu-Natal for supporting the development of this protocol and providing her technical expertise on conceptualization of this protocol. We also extend gratitude to Dr. Erigene Rutayisire and Dr. James Noah Ssemanda from Department of Public Health, Mount Kenya University, Kigali, Rwanda for technical editing earlier versions of the protocol.

Funding: No funding sources Conflict of interest: None declared

Ethical approval: The study was approved by the Institutional Ethics Committee

\section{REFERENCES}

1. Mbirimtengerenji ND. Is HIV/AIDS epidemic outcome of poverty in sub- Saharan Africa? Croat Med J. 2007;48(5):605-17.

2. Evans D, McNamara L, Maskew M, Selibas K, van Amsterdam D, Baines N, et al. Impact of nutritional supplementation on immune response, body mass index and bioelectrical impedance in HIV-positive patients starting antiretroviral therapy. Nutr J. 2013;12:111.

3. Berhe N, Tegabu D, Alemayehu M. Effect of nutritional factors on adherence to antiretroviral therapy among HIV-infected adults: a case control study in Northern Ethiopia. BMC Infect Dis. 2013; 13:233.

4. Alebel A, Wagnew F, Tesema C, Kibret GD, Petrucka P, Eshite S. Effects of undernutrition on survival of human immunodeficiency virus positive children on antiretroviral therapy. Ital $\mathrm{J}$ Pediatr. 2018;44(1):29. 
5. Duggal S, Chugh TD, Duggal AK. HIV and malnutrition: effects on immune system. Clin Dev Immunol. 2012;2012:784740.

6. Elfstrand L, Florén CH. Management of chronic diarrhea in HIV-infected patients: current treatment options, challenges and future directions. HIV AIDS (Auckl). 2010;2:219-24.

7. Arksey H, O'Malley L. Scoping studies: Towards a Methodological Framework. Int $J$ Soc Res Methodol. 2005;8:19-32.

8. Braun V, Clarke V. Using thematic analysis in psychology. Qualitative Res Psychol. 2006;3(2):77101.

9. Levac D, Colquhoun H, O'Brien KK. Scoping studies: advancing the methodology. Implementation Sci. 2010;5(1):69.

10. Hong QN, Pluye $\mathrm{P}$, Fàbregues S, Bartlett G, Boardman F, Cargo M, et al. Mixed methods appraisal tool (MMAT), version 2018. Canada; 2018.
11. Pluye P, Gagnon MP, Griffiths F, Johnson-Lafleur J. A scoring system for appraising mixed methods research, and concomitantly appraising qualitative, quantitative and mixed methods primary studies in Mixed Studies Reviews. Int J Nursing Studies. 2009;46(4):529-46.

12. Tricco AC, Lillie E, Zarin W, O'Brien KK, Colquhoun H, Levac D, et al. PRISMA extension for scoping reviews (PRISMA-ScR): checklist and explanation. Ann Internal Med. 2018;169(7):46773.

Cite this article as: Dzinamarira T, Mashora MC. Synthesizing evidence on nutrition challenges among people living with HIV/AIDS in sub- Saharan Africa: a protocol for a scoping review. Int J Community Med Public Health 2019;6:3173-6. 